\title{
Review of: "Comparison of Different Methods For Effective Manual Chest Compression In Dental Chairs, During Cardiopulmonary Resuscitation (CPR): A Manikin Study"
}

\author{
Xueke Wang ${ }^{1}$ \\ 1 Ohio State University, Columbus
}

Potential competing interests: The author(s) declared that no potential competing interests exist.

This is an interesting study that described how the different positioning of supportive tool can help reduce the vertical displacement of dental chair's backrest in CPR. The findings are with practical meaning to improve the methods used in life saving and provided useful techniques to be included in the corresponding guidelines.

1. The overall grammar and language used in this manuscript need to be improved, a lot. It's better to have a native English speaker to do some proof-reading, which can be very helpful.

2. I suggest to move the "Method" section and put it after "Introduction".

3. The last paragraph in "Discussion" can be separated to be a "Conclusion" section.

4. It's better if the authors can include some figures of the equipment (e.g. chairs, apparatus .etc.) used in this study. That will bring the readers a more intuitive perception of the differences between the testing conditions.

5. There should be better keywords that can be selected to make it clearer and better introduce this article and lure in more readers: for example "chest compression", "CPR" - this is the application of the study topic, "dental chair backrest". "Significantly" by itself doesn't make any sense to me.

6. For the data analysis method used in this study, 1) I'm interested to learn more about the details of how the vertical displacement is measured. Will that video-based measuring be accurate enough? 2) I understand that there were 600 chest compressions data collected from 3 life support providers, but how many data points did the author have to conduct that statistical analysis? What the data structure is like? It will be very helpful if the author can include a table to illustrate the different experimental conditions and data analysis approach. 